\title{
Estiércol de vacuno estabilizado y daño por boro en lechuga (Lactuca sativa L.) cv. del río, en el valle de Lluta, Chile
}

\author{
Stabilized cow manure and boron damages on lettuce (Lactuca sativa L.) \\ cv. del rio, at the Valley of Lluta, Chile
}

María E. Osorio B. ${ }^{*}$, Jorge M. Mesías M., Rodrigo Araya M.

\section{RESUMEN}

En el valle de Lluta, XV Región de Arica y Parinacota, Chile, el cultivo de lechuga (Lactuca sativa L.) cv. del río, evidencia diferencias significativas en varios factores: rendimientos, peso de las plantas, diámetros polar y ecuatorial, contenidos de nitrato y boro en las hojas; así como el daño inducido por la interacción de sales y boro, naturalmente presentes en altas concentraciones en el agua y suelos de este valle. A continuación, se informan los resultados de un estudio de mitigación de los daños inducidos por boro sobre un cultivo de lechuga (Lactuca sativa L.) cv. del río en el valle de Lluta, usando estiércol de vacuno estabilizado como agente mitigador; incluyendo un cultivo testigo, sin enmienda de estiércol y tres cultivos diferenciados con $25 \mathrm{~kg}, 50 \mathrm{~kg}$ y $100 \mathrm{~kg}$ de enmienda de estiércol de vacuno. El uso de estiércol de vacuno estabilizado en el cultivo de lechuga cv del río, produjo un significativo incremento del contenido de materia orgánica en el suelo de cultivo y una significativa aunque moderada disminución del daño inducido por los altos contenidos de boro y sales sobre las plantas de lechuga.

Palabras clave: lechuga, daños por boro, estiércol de vacuno, río Lluta, zonas áridas.

\begin{abstract}
At the Valley of Lluta, XV Region of Arica and Parinacota, Chile, the cultivation of lettuce (Lactuca sativa L.) cv. del rio, evidences significant differences in several factors: crop yields, plant weight, polar and equatorial diameters, nitrate and boron content; as well as damages induced by high concentration levels of salts and boron, naturally present in the water and cultivation soil of this valley. Here, are informed the results of a mitigation study of boron induced damages on a cultivation of lettuce (Lactuca sativa L.) cv. del rio en el valle de Lluta, using amendments of stabilized cow manure as a mitigating agent; including a witness cultivation subplot, without cow manure amendment and three cultivation subplots differentiated with $25 \mathrm{~kg}, 50 \mathrm{~kg}$ and $100 \mathrm{~kg}$ of cow manure amendments. The uses of the stabilized cow manure in the cultivation of lettuce cv del rio, yielded a significant increase in the content of organic matter in the cultivation soil and a moderate subsiding of the damages induced by the high boron and salt contents on the lettuce plants.
\end{abstract}

Key words: lettuce, damages by boron, cow manure, Lluta River, arid zones.

\section{Introducción}

Los daños inducidos por la salinidad y la toxicidad del boro (B), naturalmente presentes en altas concentraciones en el agua de riego y suelos de muchas zonas áridas y semiáridas del mundo, limitan el cultivo y explotación de especies vegetales de valor comercial, al reducir el rendimiento y calidad de los cultivos (Chávez et al., 2004). Los suelos cultivados en las zonas áridas del Norte de Chile tienen altas concentraciones de boro, así como de sales. Dichas concentraciones exceden los requerimientos nutritivos de las plantas, generando efectos tóxicos altamente dañinos para los cultivos, tal como ocurre en el valle de Lluta, XV Región de Arica y Parinacota, Chile (Figueroa et al., 1994).

El valle de Lluta se extiende entre latitudes $18^{\circ} \mathrm{S}$ y $18^{\circ} 30^{\prime} \mathrm{S}$ y entre longitudes $70^{\circ} 20^{\prime} \mathrm{O}$ y $69^{\circ} 22^{\prime} \mathrm{O}$ (Figura 1). Localizado $10 \mathrm{~km}$ al norte de la ciudad de Arica, es el valle más septentrional del país y abarca una cuenca total de drenaje de $3.378 \mathrm{~km}^{2}$,

\footnotetext{
1 Universidad de Tarapacá, Facultad de Ciencias. Agronómicas, casilla 6-D, Arica, Chile.

* Autor para correspondencia: meosorio@uta.cl
} 


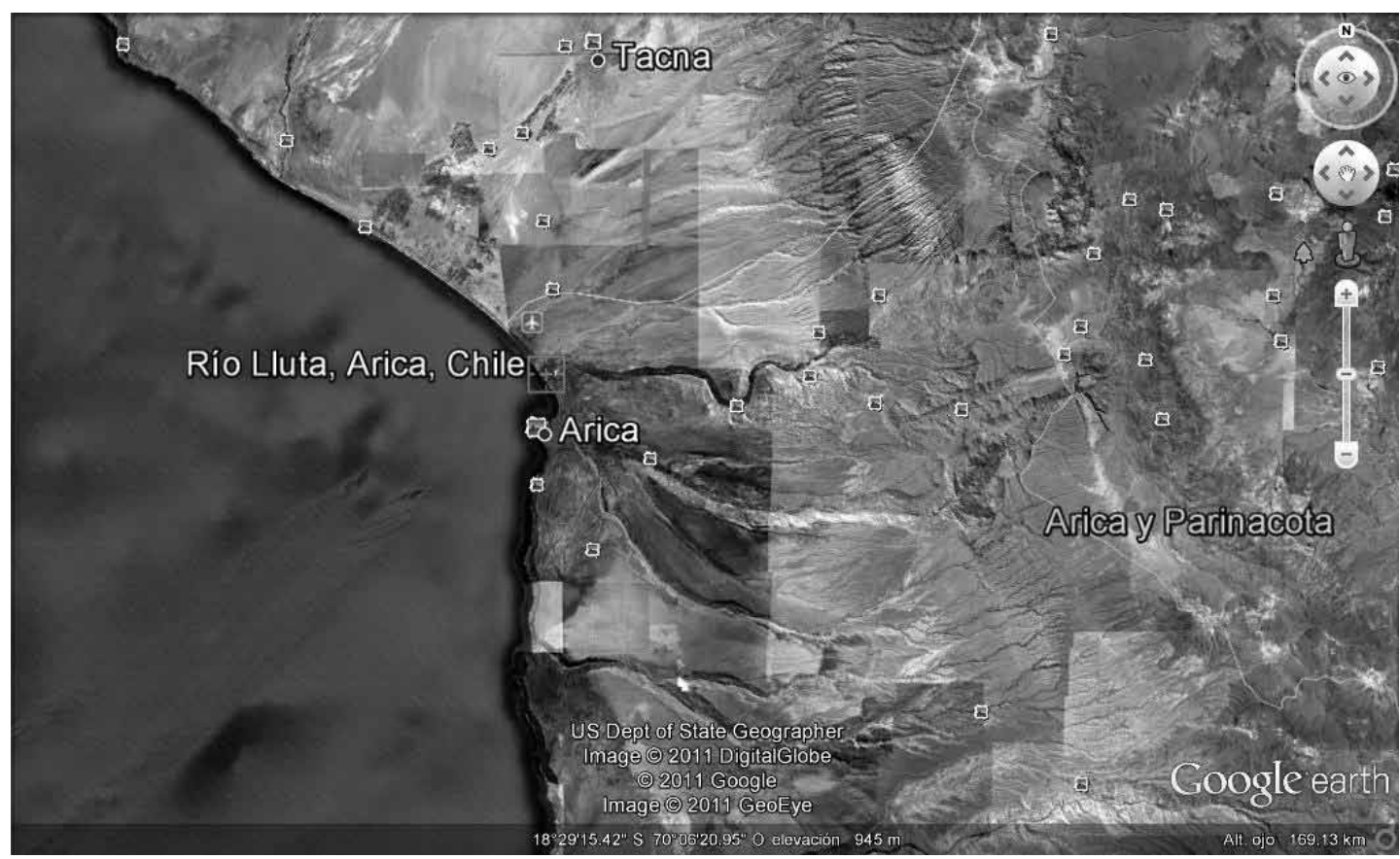

Figura 1. Cuenca del río Lluta, desde su nacimiento hasta su desembocadura. Imagen extraída usando el programa Google Earth (2012).

con una superficie agrícola potencial de 4.607 ha (MOP, 2004). Las aguas del río Lluta presentan un escurrimiento exorreico permanente en sentido Este-Oeste, que se extiende a lo largo de $65 \mathrm{~km}$ desde Vilacollo, en su extremo Este cordillerano, hasta su desembocadura en el océano Pacífico Sureste, el extremo Oeste costero de su extensión. Su principal afluente es el río Azufre; el cual al ser altamente influenciado por depósitos aluviales de origen cretácico en su recorrido rodeando el volcán Tacora y las quebradas de Caracarani, Colpitas y Socoroma, le confieren al río Lluta una alta salinidad, bajo pH y altas concentraciones de arsénico y boro (Bañuelos et al., 1999).

La cuenca del río Lluta está inserta en la zona intertropical de Sudamérica, altamente influenciada por la presencia del Anticiclón del Pacífico (Strub et al., 1998). La fuerte interacción de este último con las condiciones oceanográficas dominadas por el transporte de aguas superficiales frías desde el Sur y de surgencia desde las profundidades oceánicas costeras, inducidas por la corriente oceánica de Humboldt, definen condiciones meteorológico/climáticas de carácter desérticas para toda la zona costera que se extiende desde la zona central hasta el extremo Norte de Chile, donde se encuentra ubicado el valle del río Lluta. Así, las precipitaciones en los sectores bajos del valle (900 $\mathrm{m}$ de altitud, Poconchile) registran un promedio anual de $0,4 \mathrm{~mm}$; mientras que en las altas zonas cordilleranas ( $3.500 \mathrm{~m}$ de altitud, Putre) alcanzan un promedio anual de $237,7 \mathrm{~mm}$, presentando un régimen con máximos durante los meses estivales (diciembre a marzo), cuando el río Lluta alcanza sus máximos caudales. Debido a que el agua disponible no es suficiente para el riego de toda la superficie y el suelo no presenta condiciones de drenaje óptimas, el área efectivamente cultivada se limita a 2.784 ha, correspondientes al $37 \%$ del total de los suelos disponibles. Los cultivos se han concentrado en plantaciones de maíz (1.698 ha), alfalfa (684 ha) y sólo algunas hortalizas (402 ha); cubriendo áreas que representan el 61\%, 24,6\% y $14,44 \%$, respectivamente, del área total cultivada (MOP, 2004). Los niveles de concentración de B en el valle, varían entre los diferentes sectores desde la cordillera al mar; tanto en agua $(0,6-16 \mathrm{mg}$ $\left.\mathrm{L}^{-1}\right)$, como en el suelo $\left(0,3-24,5 \mathrm{mg} \mathrm{L}^{-1}\right)$, variando grandemente en el perfil vertical en algunas áreas específicas (Figueroa et al., 1994). 
Aunque en muchos cultivos, la toxicidad del boro se manifiesta cuando su concentración en las hojas es superior a $250-300 \mathrm{mg} \mathrm{kg}^{-1}$; la lechuga (Lactuca Sativa, L.) cv. del río posee un nivel de tolerancia moderada a concentraciones de boro en el agua de riego, de entre 1,0 y 2,05 $\mu \mathrm{gB} \mathrm{mL} \mathrm{mL}^{-1}$ (Bañuelos et al., 1999). El principal factor que influye en la adsorción y disponibilidad del boro es el pH del suelo (Goldberg, 1997); fuerte en el rango entre 3 y 9 y decrece en el rango de 10 a 11,5 (Keren et al., 1985). La toxicidad del boro disminuye también con el incremento en la concentración de $\mathrm{Ca}^{++}$(Reeve y Shive, 1944); ya que con el entrampamiento del boro se impide que éste entre en solución y sea absorbido por la planta. Además, la adsorción de boro en los suelos es mayor en suelos arcillosos que en suelos arenosos (Wear y Patterson, 1962); mientras que la biodisponibilidad y movilidad del B y elementos similares se reduce cuando decrece el contenido de humedad del suelo (Fleming, 1980). La adsorción de B decrece, también, en suelos donde predominan los minerales cristalinos (Biggar y Fireman, 1960), en el rango de temperatura entre $10^{\circ} \mathrm{C}$ y $14^{\circ} \mathrm{C}$.

Los daños inducidos por el contenido de boro sobre las plantas de cultivo son problemas comunes en muchas regiones del mundo; siendo necesaria su identificación y corrección a través de un buen conocimiento de los procesos de absorción, movilización y distribución en las plantas. Aunque está bien establecida la importancia del boro como micronutriente esencial para todas las plantas vasculares, el conocimiento de sus funciones metabólicas en los vegetales es aún incompleto (Malavé y Carreso, 2007). Históricamente, los agricultores han usado materia orgánica (MO) de diversas fuentes para mejorar sus cultivos. En los inicios de la revolución agrícola emergente en el siglo XIX, Justus Von Liebig demostró que las plantas precisan de agua y sustancias inorgánicas para su nutrición; descartando que el humus fuera el principio nutritivo de las plantas, como se hipotizaba hasta entonces. Luego, se masificó a escala global el desarrollo y uso de fertilizantes inorgánicos, ricos en nutrientes básicos como nitrógeno, fósforo y potasio, con altas concentraciones, entre 20 y 100 veces más que en los abonos orgánicos. La expansión de las prácticas agrícolas haciendo uso generalizado de fertilizantes inorgánicos, provocó el abandono de muchas técnicas previas de cultivo, tal como el abonado con residuos orgánicos (Julca-Otiniano et al., 2006).
Sin embargo, desde fines del siglo XX se ha establecido que la materia orgánica, con la que se abonan los suelos de cultivo, juega un importante rol en la recuperación de la fertilidad de los suelos sometidos a prácticas de explotación agrícola intensiva. La materia orgánica, constituida mayormente por carbono, oxígeno, hidrógeno y nitrógeno, forma parte de la fracción sólida de los suelos, íntimamente relacionada con la fracción mineral o inorgánica, formando el complejo coloidal fundamental en el intercambio catiónico y dinámica del suelo (Sierra y Rojas, 1999). Mientras que los fertilizantes inorgánicos contribuyen grandemente a acidificar o salinizar los suelos agrícolas, la materia orgánica contribuye a mantener la fertilidad de los mismos; transformando la práctica de abonar con materia orgánica en una ventaja económica para los agricultores en general. El uso de estiércol maduro de ganado en el suelo de cultivo genera condiciones de crecimiento para las plantas, mayores que las derivadas por su solo contenido de N, P y K. Esta mayor capacidad inductiva de crecimiento de las plantas se explica por el rol de microelementos contenidos en forma de quelatos en sitios de intercambio del complejo orgánico (Sierra y Rojas, 1999). El estiércol fresco de ganado vacuno presenta contenidos relativos de $\mathrm{N}(0,5 \%) ; \mathrm{P}_{2} \mathrm{O}_{5}(0,3 \%) ; \mathrm{K}_{2} \mathrm{O}(0,5 \%) ; \mathrm{Ca}(0,3 \%)$ y $\mathrm{Mg}(0,1 \%)$; mientras que en el estiércol seco, los contenidos relativos corresponden a $2,0 \% ; 1,5 \%$; $2,2 \%, 2,9 \%$ y $0,7 \%$, respectivamente. La materia orgánica humificada posee polímeros orgánicos de cadenas largas, poco accesibles a la microflora bacteriana del suelo, determinando un aporte muy bajo de nutrientes para las plantas. La materia orgánica estabilizada, sin embargo, contiene cadenas de tamaño intermedio, que pueden ser atacadas por la masa microbacteriana del suelo y representa casi el $40 \%$ del carbono total del suelo, aportando cantidades importantes de elementos nutritivos para las plantas (Sierra y Rojas, 1999, 2003).

A continuación se informan los resultados de un estudio de mitigación de los daños inducidos por boro sobre un cultivo de lechuga (Lactuca sativa L.) cv. del río en el valle de Lluta, usando estiércol de vacuno estabilizado como agente mitigador. Los resultados indican que el estiércol de vacuno jugó un rol mitigante moderado de los daños inducidos por el boro, presente en el agua de irrigación y en los suelos donde se cultivó la lechuga (Lactuca sativa L.) cv. del río. Estos resultados sugieren que una masificación del uso de estiércol de vacuno en 
las áreas de cultivo del valle de Lluta, mejoraría grandemente el rendimiento y calidad del cultivo de esta variedad de lechuga y de otras hortalizas de importancia comercial para los agricultores locales. Más aún, su masificación reduciría el uso masivo de fertilizantes inorgánicos, contribuyendo así, a limitar la emisión de gases de efecto invernadero que inducen el cambio climático global, tal como es recomendado por la Oficina de Cambio Climático del Ministerio del Medio Ambiente de Chile (Minagri, 2012).

\section{Materiales y método}

El estudio se realizó entre los meses de abril y agosto del año 2009; en condiciones abiertas al aire libre, en una superficie de $300 \mathrm{~m}^{2}$ de un predio ubicado en el km 7 del valle de Lluta (Figura 2), perteneciente a un agricultor usuario de INDAP. Para la selección del área de estudio se realizaron análisis químicos del agua, suelo y calidad del estiércol de vacuno estabilizado. Esta evaluación inicial permitió realizar todas las correcciones de cultivo correspondientes durante el estudio.

La estabilización del estiércol de vacuno se efectuó en el mes de abril, en las cercanías del lugar de estudio; mediante un proceso aeróbico controlado, considerando $1 \mathrm{~m}^{3}$ de estiércol fresco de vacuno y usando los materiales estándar para el proceso: agua, suelo estéril seco, polietileno negro, malla Rachel blanca con $60 \%$ de franjas verdes y termómetro de pincho (con $0,1^{\circ} \mathrm{C}$ de precisión) para el control de temperatura. La estabilización se realizó procurando minimizar el tamaño de partícula del estiércol, hasta alcanzar una temperatura de $70^{\circ} \mathrm{C}$ después de 30 días, óptima para ser usado como enmienda orgánica.

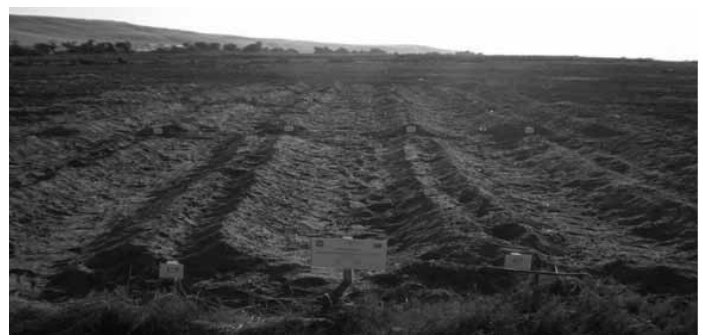

Figura 2: Imagen del terreno de cultivo en el $\mathrm{km} 7$ del valle de Lluta.

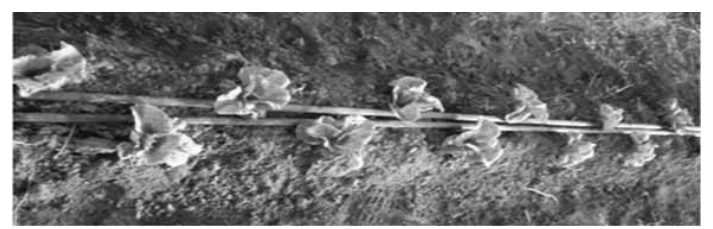

Figura 3. Ilustración de disposición de plantines de lechuga y sistema de irrigación por goteo.

El cultivo de lechuga cv. del río se estableció en el mes de mayo; estructurado en cuatro bloques aleatorios, cada uno de ellos con cuatro platabandas (para tratamientos diferenciados) y con dimensiones de 0,4 x 7,0 m cada una y distanciadas 1 m entre sí, con su respectivo sistema de riego por goteo cada una (Figura 3). En cada platabanda se dispusieron 40 plantines de lechuga cv. del río, distribuidos aleatoriamente, con separaciones de 0,4 m entre líneas y a 0,20 m sobre la línea.

El estiércol estabilizado se aplicó en dosis diferenciadas en cada platabanda. Así, se establecieron: una platabanda testigo $\left(\mathrm{T}_{0}\right)$, sin $\mathrm{MO}$; una platabanda $\left(\mathrm{T}_{1}\right)$ con tratamiento de $25 \mathrm{~kg}$ de estiércol de vacuno; una platabanda $\left(\mathrm{T}_{2}\right)$ con tratamiento de $50 \mathrm{~kg}$ de estiércol de vacuno; y una platabanda $\left(\mathrm{T}_{3}\right)$ con tratamiento de $100 \mathrm{~kg}$ de estiércol de vacuno. Cada tratamiento se repitió cuatro veces durante el período de cultivo, mecánicamente homogeneizando el suelo en cada oportunidad. El período de cultivo duró tres meses hasta la cosecha de lechugas.

Para evaluar el efecto del estiércol de vacuno; semanalmente se midieron los parámetros de $\mathrm{pH}$, conductividad eléctrica (CE), contenido de boro y materia orgánica (MO). Se tomaron tres submuestras para obtener una muestra compuesta por tratamiento seleccionado, siguiendo el método descrito por Tejeda et al., (2001). Se procuró no sobrepasar la altura de $25 \mathrm{~cm}$ en cada platabanda; tomando tres submuestras a intervalos regulares, con un peso final de 1,5 a 2,0 $\mathrm{kg}$ por muestra, después de haberse homogeneizado. Posteriormente, las muestras se llevaron a laboratorio para ser sometidas a secado y tamizado y poder tomar la respectiva alicuota (Jackson, 1982). Una vez secadas las muestras, se procedió a tamizarlas para obtener una medición del total de sales solubles (Sadzawka et al., 2004). La cuantificación del boro en el suelo se realizó por medio del método de Azometrina-H; método colorimétrico que forma un complejo coloreado, con $\mathrm{H}_{3} \mathrm{BO}_{3}$ en solución acuosa (Montoya et al., 2003). 
La medición de materia orgánica se hizo usando el método de combustión húmeda de Walkley-Black; basado en una oxidación con dicromato de potasio en medio de ácido sulfúrico. La reacción toma el calor de la disolución del ácido, lo que eleva la temperatura y logra la oxidación del carbono orgánico, para luego ser cuantificado con un espectrofotómetro de absorción atómica disponible (Manna et al., 2007).

Después de los primeros 20 días de iniciado el ensayo, se midió quincenalmente el contenido de boro en las hojas nuevas de las lechugas. El ensayo culminó con dos cosechas según su madurez y la medición de: número de hojas dañadas, peso fresco, diámetros polar y ecuatorial, daños por plagas; rendimiento en unidades y peso fresco por hectárea y por tratamiento aplicado de estiércol y contenido foliar de nitratos. De los plantines en cada platabanda se evaluaron 30 plantas, tomando una hoja por cada planta para su análisis, excluyendo las plantas de los extremos de los camellones, para evitar el efecto de los bordes. En total se evaluaron 480 plantas de las 680 distribuidas en las cuatro platabandas. Todo el ensayo se rodeó con un borde de lechugas del mismo cultivar y los tratamientos tuvieron las mismas labores culturales de riego, control de malezas y plagas, así como fertilización cuando fue necesaria para el cultivo, según análisis foliares realizados 30 días después del trasplante y según las condiciones que presentaba el suelo. Para los procesos de aplicación de estiércol y medición de parámetros se usaron: un conductivímetro WTW, modelo LF330; un medidor de pH de mesa (EXTECH 211990); un espectrofotómetro de absorción atómica (PG Instruments, modelo T211810APC) y materiales fungibles diversos.

Para evaluar el efecto del estiércol de vacuno en el rendimiento de la lechuga fue necesario esperar que éstas alcanzaran su madurez de cosecha. Las cosechas (dos) se realizaron manualmente. Luego, se determinó el peso de las unidades recolectadas, sus diámetros polar y ecuatorial, consistencia, presencia de nitratos y número de hojas dañadas. El peso fresco se obtuvo de la cabeza comercial con $0,5 \mathrm{~cm}$ de tallo al momento de cosecha. El método usado para la medición de nitrógeno total en tejido vegetal fue el de Kjeldahl. Finalmente, para determinar la evolución de la concentración iónica de boro en las hojas, las hojas preprocesadas (pesadas y secadas) fueron trituradas y calcinadas a $550^{\circ} \mathrm{C}$ por un lapso de dos horas. Luego de enfriadas, las muestras se humedecieron y se agregaron soluciones de $\mathrm{HCl}$ a 2 $\mathrm{mL}$, hasta completar el filtrado y la determinación de contenido de boro con el método de Azometrina-H.

Los resultados se analizaron mediante un análisis de varianza usando el programa Statgraphics_5.1; mientras que para las diferencias entre los promedios de los tratamientos se usó la prueba de rango múltiple de Duncan, a un nivel de confianza del 95\%. Además, se hizo uso de un programa numérico para calcular regresiones lineales siguiendo un procedimiento standard de mínimos cuadrados (Wessa, 2012), de libre disposición en Internet: www.wessa.net/slr.wasp.

\section{Resultados y discusión}

\section{Resultados iniciales previos al ensayo}

Para la selección del área de estudio se realizaron análisis químicos del agua de riego y de los suelos de cultivo; además de análisis de calidad del estiércol de vacuno estabilizado, para evaluar las condiciones iniciales de todos los tratamientos al establecer el cultivo y realizar las correcciones correspondientes.

Los resultados iniciales del análisis del agua de riego, indicaron que ésta tenía una CE de $3,1 \mathrm{dS} \mathrm{m}^{-1}$, la cual se ubica en el rango de 2,25 a 4,0 dS m${ }^{-1}$ que corresponde a la categoría $\mathrm{C} 4$, con salinidad muy alta; que en muchos casos no es apta para el riego. Esto, posiblemente es causado por el origen de las aguas del río Lluta, las que presentan altas concentraciones de sales. Para la lechuga representa una limitante, considerando que una CE de 3,4 dS $\mathrm{m}^{-1}$ en el agua de riego reduce al $50 \%$ el rendimiento de este cultivo (FAO, 1987). El pH del agua alcanzó un nivel de 6,3; mostrando que era un agua neutra con un nivel aceptable para el cultivo, ya que la lechuga tolera un $\mathrm{pH}$ entre 6,0 y 6,8 .

El contenido de calcio soluble obtenido $(280 \mathrm{mg}$ $\mathrm{L}^{-1}$ ) es muy superior al deseable entre 40 y $120 \mathrm{mg}$ $\mathrm{L}^{-1}$ en agua de riego; lo que podría provocar antagonismo con otros cationes en el suelo y manifestarse como deficiencia de alguno de ellos en el cultivo. El contenido de magnesio soluble obtenido (44,3 $\mathrm{mg} \mathrm{L}^{-1}$ ) es muy superior al nivel deseable en agua de riego (entre 6 y $24 \mathrm{mg} \mathrm{L}^{-1}$ ). El contenido de potasio obtenido (41,5 $\mathrm{mg} \mathrm{L}^{-1}$ ) es superior a lo deseable para el agua de riego (entre 0,5 y $10 \mathrm{mg} \mathrm{L}^{-1}$ ). Los contenidos obtenidos de cloruros $\left(800,5 \mathrm{mg} \mathrm{L}^{-1}\right)$ y sulfatos $\left(419,8 \mathrm{mg} \mathrm{L}^{-1}\right)$ están por sobre los deseables 
(700 $\mathrm{mg} \mathrm{L}^{-1}$ y $250 \mathrm{mg} \mathrm{L}^{-1}$, respectivamente). Los valores de sulfatos explican el bajo $\mathrm{pH}$ del agua de riego. El contenido de boro alcanzó un nivel de $28,20 \mathrm{mg} \mathrm{L}^{-1}$, característico del agua del río Lluta; representando una limitante para el cultivo de lechuga, ya que es tolerante a concentraciones entre 2 y $4,05 \mathrm{mg} \mathrm{L}^{-1} \mathrm{en}$ el agua de riego.

$\mathrm{El}$ análisis químico del suelo, previo al inicio del ensayo, mostró que la CE del suelo era de 17,33 $\mathrm{dS} \mathrm{m}^{-1}$ ), indicando un suelo altamente salino, muy superior al nivel máximo de $5 \mathrm{dS} \mathrm{m}^{-1}$, adecuado para el cultivo de lechuga (Tejeda et al., 2001). Mientras que el pH del suelo alcanzó un nivel de 6,8, aceptable para este cultivo, el contenido de materia orgánica (MO) alcanzó un nivel de 1,53\%, inferior a lo considerado como normal (entre $2,5 \%$ y $3,5 \%$ ) en suelos agrícolas. Los niveles de $\mathrm{N}\left(116,43 \mathrm{mg} \mathrm{L}^{-1}\right)$, $\mathrm{P}\left(44,47 \mathrm{mg} \mathrm{L}^{-1}\right)$ y K $\left(930,07 \mathrm{mg} \mathrm{L}^{-1}\right)$ indicaron que el suelo estaba sobrefertilizado en contenido de K. El contenido de boro del suelo alcanzó un nivel de concentración de $26,14 \mathrm{mg} \mathrm{L}^{-1}$, característico del valle de Lluta y muy superior al rango de tolerancia (2 a $4 \mathrm{mg} \mathrm{L}^{-1}$ ) de la lechuga cv. del río.

En las muestras de suelo, además, se identificaron 10 ejemplares de nematodos, de los géneros Cephalobus sp. (9) y Dorylaimida sp. (1); los cuales no tienen incidencia en la sanidad del cultivo de lechuga; sin encontrar nematodos fitoparásitos como Meloidogyne arenania, los que usualmente atacan cultivos hortícolas como la lechuga cv. del río en suelos con bajos contenidos de materia orgánica (Kratochvil et al., 2004). Estos resultados iniciales previos al ensayo permitieron establecer que no era necesaria la incorporación de fertilizantes químicos como abonado de fondo; siendo éstos incorporados sólo de acuerdo al análisis foliar realizado después de iniciado el ensayo.

En el análisis químico del estiércol de vacuno estabilizado se encontraron niveles de CE (6,69 dS $\left.\mathrm{m}^{-1}\right) ; \mathrm{pH}(7,45) ; \mathrm{MO}(25,5 \%) ; \mathrm{N}$ total $(1,20 \%) ; \mathrm{P}$ asimilable $\left(\mathrm{P}_{2} \mathrm{O}_{5}, 0,65 \%\right)$; potasio $\left(\mathrm{K}_{2} \mathrm{O}, 2,10 \%\right)$; calcio ( $\mathrm{Ca} \mathrm{O}, 2,14 \%)$; magnesio $(\mathrm{Mg} \mathrm{O}, 0,51 \%)$; y boro soluble $\left(19,2 \mathrm{mg} \mathrm{L}^{-1}\right)$. Estos resultados indicaron que el estiércol de vacuno estaba en condiciones maduras, con un alto contenido de sales y boro para el cultivo de lechuga cv. del río; sin embargo, el contenido de boro soluble es aceptable, considerando que se obtuvo de los alrededores del ensayo, lo cual indica que estos animales beben el agua del río Lluta y son alimentados con cultivos propios del lugar.

\section{Resultados de los tratamientos}

A continuación se presentan separadamente los resultados de las mediciones caracterizando las propiedades del suelo de cultivo (A), de aquellos que caracterizan las plantas de lechuga cosechadas de los diferentes tratamientos (B).

\section{A. Comportamiento del suelo de cultivo}

Los resultados del comportamiento del suelo de cultivo, a los tratamientos diferenciados con estiércol de vacuno estabilizado, se presentan resumidos en la Tabla I:

\section{A.1 Comportamiento del contenido de materia orgánica (MO) del suelo del cultivo}

En la Tabla I, fila 1, se muestra el contenido de MO del suelo, que exhibe una relación lineal positiva creciente con la cantidad de estiércol de vacuno aplicado o tratamiento $(\mathrm{T})$; con un coeficiente de correlación que explica hasta el $61 \%$ de su varianza y casi nula autocorrelación. Los análisis de varianza de las mediciones en cada platabanda indicaron que existen diferencias significativas entre bloques así como en los tratamientos, con un coeficiente de variabilidad de $12,87 \%$, aceptable para las condiciones del experimento. Este resultado indica que dosis crecientes de aplicaciones de estiércol de vacuno incrementan significativamente el contenido de MO en los suelos de cultivo.

\section{A.2 Comportamiento del $\mathrm{pH}$ del suelo de cultivo}

En la Tabla I, fila 2, se muestran los promedios temporales de $\mathrm{pH}$ del suelo, obtenidos desde el inicio del ensayo. Estos promedios temporales muestran una relación lineal positiva con la cantidad de estiércol de vacuno aplicado o tratamiento $(\mathrm{T})$; con un coeficiente de correlación que explica hasta el $28 \%$ de su varianza y casi nula autocorrelación. Los análisis de varianza de las mediciones en cada platabanda indicaron que no existen diferencias significativas entre bloques ni entre tratamientos (bloques homogéneos), con un coeficiente de variabilidad de $5,17 \%$, aceptable para las condiciones del ensayo.

Estos resultados indican que aplicaciones de dosis crecientes de estiércol de vacuno aumentan significativamente el $\mathrm{pH}$ de los suelos de cultivo. 
Tabla I. Comportamiento del suelo de cultivo.

\begin{tabular}{|c|c|c|c|c|c|c|c|c|c|}
\hline Indice & $\begin{array}{l}\text { Variable } \\
\text { (unidad) }\end{array}$ & $\begin{array}{c}\mathbf{T}_{0} \\
(\mathbf{0} \\
\mathbf{k g E V})\end{array}$ & $\begin{array}{c}T_{1} \\
(25 \\
\text { kgEV) }\end{array}$ & $\begin{array}{c}T_{2} \\
(\mathbf{5 0} \\
\operatorname{kgEV})\end{array}$ & $\begin{array}{c}T_{3} \\
(100 \\
\text { kgEV })\end{array}$ & $\begin{array}{c}\mathbf{Y}_{0} \\
\text { constante }\end{array}$ & $\begin{array}{c}\beta \\
\text { pendiente }\end{array}$ & $Q^{2}$ & $\begin{array}{c}\varrho \\
\text { auto- } \\
\text { correlación }\end{array}$ \\
\hline 1 & $\begin{array}{l}\text { MO } \\
(\%)\end{array}$ & 1,5 & 2,2 & 3,1 & 4,7 & 1,46 & 0,03 & $-0,61$ & $-0,81$ \\
\hline 2 & $\underset{(\text { ) }}{\mathbf{p H}}$ & 5,8 & 6,6 & 6,9 & 7,1 & 6,08 & 0,01 & $-0,28$ & $-0,32$ \\
\hline 3 & $\begin{array}{c}\mathbf{C E} \\
\left(\mathrm{dS} \mathrm{\textrm {m } ^ { - 1 }}\right)\end{array}$ & 9,1 & 6,6 & 5,4 & 4,1 & 8,36 & $-0,05$ & $-0,28$ & $-0,32$ \\
\hline 4 & $\underset{\left(\mathrm{mg} \mathrm{L}^{-1}\right)}{\mathbf{B}}$ & 24,3 & 26,7 & 31,0 & 35,1 & 24,4 & 0,11 & $-0,64$ & $-0,76$ \\
\hline
\end{tabular}

FILAS: 1. MO, materia orgánica; 2. pH; 3. CE, conductividad eléctrica; 4. B, boro; COLUMNA: T 0 , tratamiento testigo sin estiércol de vacuno; $\mathbf{T}_{1}$, tratamiento con $25 \mathrm{kgEV} /$ platabanda; $\mathbf{T}_{2}$, tratamiento con $50 \mathrm{kgEV} /$ platabanda; y $\mathbf{T}_{3}$, tratamiento con $100 \mathrm{kgEV} /$ platabanda (donde EV significa estiércol de vacuno). $\mathbf{Y}_{0}$ y $\beta$ representan la constante y pendiente, respectivamente, de regresiones lineales independientes de cada variable $(\mathbf{Y})$ del suelo versus los tratamientos $(\mathbf{T})$ con estiércol de vacuno, de la forma: $\mathbf{Y}=\mathbf{Y}_{0}+\beta \mathbf{T} ; \varrho^{2}$ y $\varrho$ (autocorrelación), representan coeficientes de correlación para cada regresión lineal calculada. Los valores de: MO, pH, CE y B, filas 1 a 4, respectivamente, representan promedios temporales de mediciones hechas cada siete días (10 en total) en cada platabanda.

Sin embargo, el tratamiento $\mathrm{T}_{0}(\sin \mathrm{MO})$ presenta mayor variabilidad en $\mathrm{pH}$; reafirmando que la materia orgánica actúa como agente tampón, al disminuir la tendencia a cambios bruscos de $\mathrm{pH}$ del suelo, cuando se aplican sustancias de reacción ácida o alcalina (Munita, 2001). La mayor variabilidad mostrada en el tratamiento $\mathrm{T}_{0}$ puede deberse a cambios en la calidad del agua de riego (asociados a su variabilidad estacional).

\section{A.3 Comportamiento de la conductividad eléctrica}

(CE) del suelo de cultivo

En la Tabla I, Fila 3, se muestran los promedios temporales de la CE del suelo para las diferentes platabandas; obtenidos promediando las 10 mediciones realizadas cada siete días, desde el inicio del ensayo. Estos promedios temporales muestran una relación lineal negativa con la cantidad de estiércol de vacuno aplicado o tratamiento (T); con un coeficiente de correlación que explica hasta el $28 \%$ de su varianza y casi nula autocorrelación. Los análisis de varianza de las mediciones en cada platabanda indicaron que no existen diferencias significativas entre bloques (bloques homogéneos), pero sí entre tratamientos, con un coeficiente de variabilidad de $19,8 \%$, aceptable para las condiciones del estudio. Esta relación inversa entre CE y tratamientos (T) reafirma que la incorporación de estiércol de vacuno (y MO) al suelo proporciona una protección efectiva contra los efectos adversos de las sales, lo que concuerda con resultados previos informando que suelos más fértiles contienen menos cantidades de sales solubles (USDA, 1996).

A.4 Comportamiento del contenido de boro del suelo de cultivo

En la Tabla I, fila 4, se muestran los contenidos de boro del suelo, obtenidos promediando todas las (10) mediciones para cada platabanda, hechas cada siete días desde el inicio del ensayo. Estos promedios temporales muestran una relación lineal positiva creciente con la cantidad de estiércol de vacuno aplicado o tratamiento (T); con un coeficiente de correlación que explica hasta el $64 \%$ de su varianza y casi nula autocorrelación. Los análisis de varianza de las mediciones en cada platabanda indicaron que no existen diferencias significativas entre bloques (bloques homogéneos), pero sí entre tratamientos, con un coeficiente de variabilidad de 7,19\%, aceptable para las condiciones del estudio. Este resultado indica que dosis crecientes de enmiendas de estiércol de vacuno incrementan significativamente el contenido de MO en los suelos de cultivo. Estos resultados muestran que la MO fija grandes cantidades de boro en los suelos, lo que concuerda con resultados de previas investigaciones (Parks 
y White, 1952). La disminución del contenido de boro se produjo paralelamente a la variación del contenido del mismo en el agua de riego, confirmando la variabilidad estacional del contenido de sales del agua del río Lluta.

\section{B. Comportamiento de las plantas cultivadas}

Los resultados del comportamiento de las plantas de lechuga (Lactuca sativa L.) cv. del río, cultivadas con tratamiento diferenciado de estiércol de vacuno estabilizado, se presentan resumidos en las Tablas II y III. En la Tabla II se resumen los resultados del comportamiento de los minerales en las hojas de lechuga; mientras que en la Tabla III se resumen los resultados que caracterizan el daño inducido por boro y sales sobre las plantas de lechuga.

\section{B.1. Comportamiento del contenido de boro en hojas de lechugas}

En la Tabla II, fila 1, se muestran los promedios temporales del contenido de B en las hojas de las plantas de lechuga cultivadas en cada platabanda. Estos promedios temporales muestran una relación lineal positiva creciente con la cantidad de estiércol de vacuno aplicado o tratamiento (T); con un coeficiente de correlación que explica hasta el $27 \%$ de su varianza y casi nula autocorrelación. Los análisis de varianza de las mediciones en cada platabanda indicaron que no existen diferencias significativas entre bloques (bloques homogéneos), pero sí entre tratamientos, con un coeficiente de variabilidad de $2,79 \%$, aceptable para las condiciones del experimento. Estos resultados indican que dosis crecientes de aplicaciones de estiércol de vacuno disminuyeron significativamente la toxicidad por B, aunque el contenido de B alcanzado en las plantas supera altamente los niveles de tolerancia (250 a $300 \mathrm{mg} \mathrm{kg}^{-1}$.) de las mismas. Estos resultados concuerdan con resultados previos (Yermiyahu et al., 2001) que reafirman que la aplicación de MO a suelos con altos contenido de boro disminuyen y/o evitan la toxicidad de la planta.

\section{B.2. Comportamiento del contenido de nitratos en hojas de lechugas}

En la Tabla II, fila 2, se muestran los contenidos de nitratos $\left(\mathrm{NO}_{3}\right)$ de las hojas de lechuga cosechadas en cada platabanda, los que muestran una relación lineal positiva creciente con la cantidad de estiércol de vacuno aplicado o tratamiento (T); con un coeficiente de correlación que explica hasta el 35\% de su varianza y casi nula autocorrelación. Los análisis de varianza de las mediciones en cada platabanda indicaron que no existen diferencias significativas entre bloques (bloques homogéneos), pero sí entre tratamientos, con un coeficiente de variabilidad de $2,61 \%$, aceptable para las condiciones del experimento.

Estos resultados muestran que dosis crecientes de aplicaciones de estiércol de vacuno aumentan significativamente los contenidos de nitratos en hojas, por lo que deben evitarse grandes dosis de estiércol en los cultivos de lechuga, pues representan grandes riesgos para la salud humana. Sin embargo, los tratamientos están dentro de los limites máximos permisibles de 3.500 a $4.000 \mathrm{mg} \mathrm{kg}^{-1}$, en Alemania y Suiza (Santamaría, 2006).

B.3. Comportamiento de los contenidos de fósforo, calcio, potasio, sodio y nitrógeno en hojas de lechugas

Los contenidos de fósforo, calcio, potasio, sodio y nitrógeno en las hojas de lechuga cosechadas que se muestran en la Tabla II, filas 3, 4 y 5 , respectivamente, muestran casi nulas relaciones lineales con los tratamientos ( $\mathrm{T}$ ); con coeficientes de correlación que explican hasta el 33\%, 28\% y $48 \%$, respectivamente, de sus varianzas y con casi nulas autocorrelaciones. En contraste, los contenidos de sodio y nitrógeno, filas 6 y 7 , respectivamente, muestran una clara relación lineal positiva, con coeficientes de correlación que explican hasta el $41 \%$ y $34 \%$, respectivamente, de sus varianzas. Esto significa que la aplicación de estiércol de vacuno no produce un cambio significativo en los contenidos de fósforo, calcio y potasio, pero sí introduce incrementos significativos en los contenidos de sodio y nitrógeno.

B.4. Comportamiento del peso fresco y diámetros ecuatorial y polar de las plantas de lechugas cosechadas

Los valores de peso fresco (Pf) y diámetros ecuatorial (De) y polar (Dp) de las plantas de lechuga cosechadas en las platabandas se muestran en la Tabla III, filas 1, 2 y 3, respectivamente. Los resultados muestran una relación lineal positiva con 
Tabla II. Comportamiento de minerales en las hojas de las plantas cultivadas.

\begin{tabular}{|c|c|c|c|c|c|c|c|c|c|}
\hline Índice & $\begin{array}{l}\text { Variable } \\
\text { (unidad) }\end{array}$ & $\begin{array}{c}\mathbf{T}_{0} \\
(\mathbf{0} \\
\operatorname{kgEV})\end{array}$ & $\begin{array}{c}\mathrm{T}_{1} \\
(25 \\
\mathrm{kgEV})\end{array}$ & $\begin{array}{c}T_{2} \\
(50 \\
\operatorname{kgEV})\end{array}$ & $\begin{array}{c}T_{3} \\
(100 \\
\operatorname{kgEV})\end{array}$ & $\underset{\text { constante }}{\mathbf{Y}_{0}}$ & $\begin{array}{c}\beta \\
\text { pendiente }\end{array}$ & $\varrho^{2}$ & $\begin{array}{c}\varrho \\
\text { auto- } \\
\text { correlación }\end{array}$ \\
\hline 1 & $\begin{array}{c}\text { B } \\
\left(\mathrm{mg} \mathrm{kg}^{-1}\right)\end{array}$ & 510,0 & 457,4 & 423,5 & 387,00 & 496,26 & $-1,18$ & $-0,27$ & $-0,31$ \\
\hline 2 & $\underset{\left(\mathrm{mg} \mathrm{kg}^{-1}\right)}{\mathbf{N O}_{3}}$ & 598,50 & 639,00 & 681,20 & 711,40 & 608,66 & 1,12 & $-0,35$ & $-0,38$ \\
\hline 3 & $\begin{array}{c}\mathbf{P} \\
(\%)\end{array}$ & 0,30 & 0,50 & 0,50 & 0,50 & 0,38 & $-0,01$ & $-0,33$ & $-0,40$ \\
\hline 4 & $\begin{array}{c}\mathbf{C a}^{++} \\
(\%)\end{array}$ & 0,80 & 1,20 & 1,30 & 1,30 & 0,96 & 0,001 & $-0,28$ & $-0,33$ \\
\hline 5 & $\underset{(\%)}{\mathbf{K}}$ & 2,70 & 2,80 & 3,00 & 3,00 & 2,74 & 0,01 & $-0,48$ & $-0,54$ \\
\hline 6 & $\begin{array}{c}\mathbf{N a} \\
\left(\mathrm{mg} \mathrm{kg}^{-1}\right)\end{array}$ & 6319,80 & 9040,90 & 9202,30 & 10554,70 & 7137,80 & 37,52 & $-0,41$ & $-0,5$ \\
\hline 7 & $\underset{\left(\mathrm{mg} \mathrm{kg}^{-1}\right)}{\mathbf{N}}$ & 27033,90 & 28863,70 & 30768,40 & 32134,00 & 27492,70 & 50,45 & $-0,34$ & $-0,38$ \\
\hline
\end{tabular}

FILAS: 1. B, promedios temporales de mediciones cada siete días (10 en total) hechas en cada platabanda de contenido de boro en hojas; 2. NO ${ }_{3}$, contenido de nitratos en hojas: 3. P, contenido de fósforo en hojas; $\mathbf{4}$. $\mathbf{C a}^{++}$, contenido de calcio en hojas; 5. K, contenido de potasio en hojas; $\mathbf{6}$. Na, contenido de sodio en hojas; $\mathbf{7}, \mathbf{N}$, contenido de nitrógeno en hojas; COLUMNA: $\mathbf{T}_{0}$, tratamiento testigo sin estiércol de vacuno; $\mathbf{T}_{1}$, tratamiento con $25 \mathrm{kgEV} /$ platabanda; $\mathbf{T}_{2}$, tratamiento con $50 \mathrm{kgEV} / \mathrm{platabanda}$; $\mathbf{y} \mathbf{T}_{3}$, tratamiento con $100 \mathrm{kgEV} /$ platabanda (donde EV significa estiércol de vacuno). $\mathbf{Y}_{0}$ y $\beta$ representan la constante y pendiente, respectivamente, de regresiones lineales independientes de cada variable $(\mathrm{Y})$ del suelo versus los tratamientos $(\mathrm{T})$ con estiércol de vacuno, de la forma: $\mathbf{Y}=\mathbf{Y}_{0}+\beta \mathrm{T} ; \varrho^{2}$ y $\varrho$ (autocorrelación), representan coeficientes de correlación para cada regresión lineal calculada.

Tabla III. Comportamiento del daño en las plantas cultivadas.

\begin{tabular}{|c|c|c|c|c|c|c|c|c|c|}
\hline Índice & $\begin{array}{l}\text { Variable } \\
\text { (unidad) }\end{array}$ & $\begin{array}{c}\mathbf{T}_{0} \\
(\mathbf{0} \\
\mathbf{k g E V})\end{array}$ & $\begin{array}{c}T_{1} \\
(25 \\
\text { kgEV) }\end{array}$ & $\begin{array}{c}T_{2} \\
(\mathbf{5 0} \\
\mathbf{k g E V})\end{array}$ & $\begin{array}{c}T_{3} \\
(100 \\
\operatorname{kgEV})\end{array}$ & $\underset{\text { constante }}{\mathbf{Y}_{0}}$ & $\begin{array}{c}\beta \\
\text { pendiente }\end{array}$ & $\varrho^{2}$ & $\begin{array}{c}Q \\
\text { auto- } \\
\text { correlación }\end{array}$ \\
\hline 1 & $\begin{array}{l}\text { Pf } \\
(\mathrm{g})\end{array}$ & 341,20 & 485,20 & 662,40 & 702,80 & 390,72 & 3,59 & $-0,38$ & $-0,42$ \\
\hline 2 & $\begin{array}{l}\text { De } \\
(\mathrm{cm})\end{array}$ & 17,30 & 20,40 & 23,40 & 24,60 & 18,30 & 0,071 & $-0,32$ & $-0,36$ \\
\hline 3 & $\begin{array}{l}\text { Dp } \\
(\mathrm{cm})\end{array}$ & 12,40 & 15,10 & 17,90 & 18,60 & 13,34 & 0,06 & $-0,34$ & $-0,37$ \\
\hline 4 & $\begin{array}{l}\text { Nhd } \\
\text { ( ) }\end{array}$ & 15 & 14 & 11 & 10 & 14,80 & $-0,052$ & $-0,62$ & $-0,73$ \\
\hline 5 & $\begin{array}{l}\text { Nhpc } \\
\text { ( ) }\end{array}$ & 35,20 & 37,40 & 39,80 & 40,30 & 35,98 & 0,03 & $-0,35$ & $-0,39$ \\
\hline 6 & $\begin{array}{l}\text { Npb } \\
\text { ( ) }\end{array}$ & 17 & 10,25 & 2,50 & 1,50 & 14,50 & $-0,15$ & -0.36 & -0.40 \\
\hline 7 & $\begin{array}{l}\text { Npf } \\
()\end{array}$ & 9,30 & 11,50 & 7,00 & 5,80 & 5,80 & $-0,04$ & $-0,66$ & -0.88 \\
\hline
\end{tabular}

FILAS: 1. Pf, peso fresco de plantas; 2. De, diámetro ecuatorial de plantas; 3. Dp, diámetro polar de plantas; 4. Nhd, número de hojas dañadas; 5. Nhpc, número de hojas por planta completa; 6 . Npb, número de plantas blandas; 7. Npf, número de plantas firmes; COLUMNA: $\mathbf{T}_{0}$, tratamiento testigo sin estiércol de vacuno; $\mathbf{T}_{1}$, tratamiento con $25 \mathrm{~kg}$ de estiércol de vacuno; $\mathbf{T}_{2}$, tratamiento con $50 \mathrm{~kg}$ de estiércol de vacuno; y $\mathbf{T}_{3}$, tratamiento con $100 \mathrm{~kg}$ de estiércol de vacuno. $\mathbf{Y}_{0}$ y $\beta$ representan la constante y pendiente, respectivamente, de regresiones lineales independientes de cada variable $(\mathrm{Y})$ del suelo versus los tratamientos $(\mathrm{T})$ con estiércol de vacuno, de la forma: $\mathbf{Y}=\mathbf{Y}_{0}+\beta \mathbf{T} ; \varrho^{2}$ y $\varrho$ (autocorrelación) representan coeficientes de correlación para cada regresión lineal calculada. 
los tratamientos, con coeficientes de correlación que explican hasta el $38 \%, 32 \%$ y el $34 \%$, respectivamente, de sus varianzas y casi nulas autocorrelaciones. Esto significa que el peso fresco de lechuga y sus diámetros ecuatorial y polar aumentan en forma creciente a medida que aumenta el contenido de materia orgánica incorporada al suelo de cultivo.

\section{B.5.Daños en hojas por boro y sales en las plantas}

En las filas 4 a 7 de la Tabla III se muestran, respectivamente: el número de hojas dañadas por cada planta (Nhd), el número de hojas por cada planta completa (Nhpc), el número de plantas blandas ( $\mathrm{Npb}$ ) y el número de plantas firmes (Npf), medidas de las plantas de lechuga cosechadas. Los resultados muestran una relación lineal negativa para Nhd, con un coeficiente de correlación que explica hasta el $62 \%$ de su varianza y casi nula autocorrelación; implicando directamente una disminución del daño inducido por boro; a medida que se incrementa el contenido de materia orgánica incorporada al suelo de cultivo. Los valores de Nhpc, Npb y Npf presentan una débil relación lineal, con coeficientes de correlación que explican hasta el 35\%, 36\% y $66 \%$ de sus varianzas respectivas, pero teniendo todas casi nula autocorrelación. Estos resultados implican que mientras aumenta significativamente el número de hojas por cada planta completa, los daños inducidos por boro decrecen a medida que se incorporan mayores dosis de estiércol de vacuno al suelo de cultivo.

\section{Conclusiones}

De los resultados obtenidos con mediciones que caracterizan el suelo de cultivo podemos concluir que la incorporación de estiércol de vacuno al suelo determina un aumento lineal de la materia orgánica (MO) del mismo. La MO juega un papel importante como agente tampón, evitando bruscas variaciones del $\mathrm{pH}$ en el suelo; mientras se origina una disminución de la $\mathrm{CE}$, por una mayor fijación de boro en el suelo.
De los resultados obtenidos con las mediciones que caracterizan las plantas de lechuga cosechadas de las platabandas de ensayo, podemos concluir que la aplicación de estiércol de vacuno en dosis crecientes aumentan el rendimiento en peso unitario del cultivo de lechuga, al mismo tiempo que disminuyen moderadamente los daños inducidos por el boro contenido en las aguas de riego y en los suelos de cultivo.

Así, podemos concluir que es recomendable el uso de estiércol de vacuno estabilizado para incrementar la materia orgánica en los suelos de cultivo en el valle del río Lluta, previa realización de análisis químicos del suelo y agua de riego, antes de establecer cualquier cultivo, para evaluar la necesidad de un abonado de fondo y evitar los riesgos de sobrefertilización química que determinen un aumento del contenido de sales y contaminación de los suelos de cultivo.

Se recomienda investigar el efecto de la interacción boro-sales en el mismo cultivo de lechuga (Lactuca sativa L.) cv. del río; pues en los análisis practicados a estos suelos, los contenidos de sales fueron excesivos y tanto el daño en hojas como el menor rendimiento observado en los diferentes tratamientos, no pueden ser atribuidos exclusivamente al alto contenido de boro en los suelos del valle de Lluta.

\section{Agradecimientos}

Agradecemos al Sr. Luis González Silva, propietario del predio agrícola del valle de Lluta usado en este estudio. También agradecemos a Lic. Patricia Pacheco C. por su contribución en nuestro programa de mediciones en el laboratorio de suelos y aguas, FADECIA, UTA. Particularmente, agradecemos a la Dra. Claudia Silva por una cuidadosa revisión del manuscrito original y sus comentarios sobre la versión final del mismo. Finalmente, agradecemos también al Dr. P. Wessa por disponer gratuitamente al público en internet de su programa computacional estadístico (Free Statistics Software), usado intensivamente en nuestros cálculos de regresiones lineales numéricas. 


\section{Literatura Citada}

Bañuelos, G.; Ajwa, H.; Cáceres, L.; Dyer, D.

1999. Diferencias de acumulación y tolerancia a altas concentraciones de boro de especies agrícolas entre el Norte de Chile y California, Estados Unidos. Idesia (17): 31-40.

Biggar W.J.; Fireman, M.

1960. Boron adsorption and release by soils. Soil Sci. Soc. Amer. Proc. 24: 115-120.

Chávez, R.; Mery Aduciri, M.; Linares, J.; García, A.

2004. Respuestas fenotípicas de híbridos y variedades de maíz (Zea mays L.) al estrés de salinidad y toxicidad de boro bajo condiciones de laboratorio y campo. Idesia 22 (2): 11-28.

Figueroa, L.; Tapia, L.; Bastías, E.; Escobar, H.; Torres, A. 1994. Niveles de Boro en aguas de riego y suelos que sustentan olivos en el Norte de Chile. Idesia 13: 63-68.

Fleming, G.

1980. Essential micronutrients, I: Boron and molybdenum. In Applied Soil Trace. Ed. Davis: 155-197.

Goldberg, S.

1997. Reaction of boron with soils. Plant and Soil Proc. 193: 35-48.

Jackson, M.L.

1982. Análisis químico de suelos. Barcelona, España. 4 Ed. Omega. 662 p.

Julca-Otiniano, A.; Meneses-Florian, L.; Blas-Sevillano, R.;

Bello-Amez, S.

2006. La materia orgánica, importancia y experiencias de su uso en la agricultura. Idesia 24 (1): 49-26.

Keren, R.; Bingham, F.T.; Rhoades, J.D.

1985. Plant uptake of boron as affected by boron distribution between liquid and solid phases in soil. Soil Sci. Soc. Am. J. 49: 297-302.

Kratochvil, R.J.; Sardanelli, S.; Everts, K.; Gallagher, E.

2004. Evaluation of crop rotation and other cultural practices for management of root-knot lesion nematodes. Agronomy J. 96 (5): 1419-1428.

Malave, A.; Carreso, P.

2007. Desempeño funcional del boro en las plantas. Rev. Cient. UDO agrícola 7 (1): 1-14.

Manna, T.; Honnappa, S.; Steinmetz, M.O.; Wilson, L.

2007. Suppression of microtubule dynamic instability by the +TIP protein EB1 and its modulation by the CAP-Gly domain of p150 (Glued). Biochemistry. 47: 779-786.

Ministerio de Obras Públicas (MOP): Dirección General de Aguas.

2004. Diagnóstico y clasificación de los cursos y cuerpos de agua según objetivos de calidad. Cuenca del río Lluta.
Ministerio de Agricultura (Minagri).

2012. Propuesta Plan de adaptación al cambio climático del sector silvoagropecuario, Minagri 2352012.doc.

Montoya, J.M.; Rodríguez, M.A.; Hawkins B.A.

2003. Food web complexity and higher-level ecosystem services. Ecology Letters 6: 587-593.

Munita, J.

2001. Características y clasificación de los suelos. Agenda del Salitre, Soc. Quím. Min. de Chile. pp. 27-50.

Parks, W.L.; White, J.L.

1952. Boron retention by clay and humus systems saturated with various cations. Soil Sci. Soc. Am. Proc. 16: 298-300.

Reeve, E.; Shive, W.

1944. Potassium-boron and calcium-boron relationship on plant nutrition. Soil Sci. Proc. 57: 1-14.

Sadzawka, A.M.; Carrasco, M.A.; Grez, R.; Mora, M.L.

2004. Métodos de análisis recomendados para los suelos chilenos. Comisión de normalización y acreditación. Soc. Ch. de la Ciencia del Suelo. Santiago, Chile, 113 p.

Santamaría, P.

2006. Nitrate in vegetables: toxicity, content, intake and EC regulation. J. Sci. Food Agric. 86:10-17.

Sierra, C.; Rojas, C.

1999. Materia orgánica del suelo, naturaleza, mantención y su importancia en la agricultura. Serie Remehue, 71: 16-56.

Sierra, C.; Rojas, C.

2003. La materia orgánica y su efecto en las características físico-químicas y biológicas del suelo. Serie Remehue, 71: $5-22$.

Strub, P.T.; Mesias, J.M.; Montecino, V.; Rutllant, J.; Salinas, S. 1998. Coastal ocean circulation off western South America, The Sea, 11, A. R. Robinson and K. H. Brink (Eds.): 273-313.

Tejeda, H.; Sadzawka, M.; Araos, J.

2001. Análisis de suelo, foliar y de agua para el diagnóstico y uso de fertilizantes y enmiendas. Agenda del Salitre, Soc. Quím. Min. de Chile. p. 187-230.

Wear, J.; Patterson, P.

1962. Effect of soil $\mathrm{pH}$ and texture on the availability of water soluble boron in the soil. Soil Sci. Society of Am. Proc. 26 (4): $344-346$

Wessa, P.

2012. Free Statistics Software, Office for Research Development and Education, version 1.1.23-r7, URL http:// www.wessa. net/

Yermiyahu, U.; Keren, R.; Chen, R.

2001. Effect of composted organic matter on boron uptake by plants. Soil Sci. of Am. J. 65 (5): 1436-1441. 
